\title{
Comparison of the effects of subinhibitory concentrations of ciprofloxacin and colistin on the morphology of cardiolipin domains in Escherichia coli membranes \\ Correspondence \\ Andrzej B. Hendrich \\ andrzej.hendrich@am.wroc.pl \\ Received 31 August 2011 \\ Accepted 2 December 2011 \\ Marta Kicia, Natalia Janeczko, Jagoda Lewicka and Andrzej B. Hendrich \\ Department of Medical Biology and Parasitology, Wrocław Medical University, ul. Mikulicza-Radeckiego 9, 50-367 Wrocław, Poland

\begin{abstract}
Membrane domains characterized by unique protein and lipid composition allow for compartmentalization and regulation of various biological processes. In Escherichia coli cardiolipin domains play a key role in the dynamic organization of bacterial membranes, and their distribution depends on the stage of the cell cycle. We studied the influence of subinhibitory concentrations of ciprofloxacin and colistin on the morphology and distribution of $E$. coli cardiolipin domains. Using the fluorescent dye 10-N-nonyl acridine orange we found that exposure of bacteria to ciprofloxacin significantly increased the percentage of filamentous cells with altered morphology of the cardiolipin domains, while colistin did not induce any significant changes. These results allow us to conclude that inhibition of DNA gyrase causes effects even at the bacterial membrane level and those changes can be easily visualized using $10-N$-nonyl acridine orange.
\end{abstract}

\section{INTRODUCTION}

Forty years after the introduction of the fluid mosaic model of the cell membrane, the heterogeneity of membranes is acknowledged as one of the most important aspects of this model. The role of membrane domains and/or lipid rafts is being increasingly appreciated among membrane scientists. The concept of the presence of lipid domains is also valid for bacterial membranes, whose lipid composition (mainly phosphatidylglycerol and cardiolipin; also phosphatidylethanolamine, phosphatidylserine and phosphatidic acid: Tucker \& White, 1970) is different from that of eukaryotic membranes. In recent years a simple but efficient method enabling certain aspects of the bacterial cell membrane domain structure to be followed has been proposed (Mileykovskaya \& Dowhan, 2000). The principle of this method is based on the unique properties of the fluorescent label $10-\mathrm{N}$-nonyl acridine orange (NAO). This dye binds preferentially to anionic lipids present in bacterial membranes and thus enables the membrane distribution of these lipids to be observed. What is even more important, binding of NAO with cardiolipin (CL, which can be considered to some extent as a phosphatidylglycerol dimer) changes the spectral properties of this dye by shifting its fluorescence from green to red (Petit et al., 1992). This special feature of NAO, as well as its much higher affinity for CL over monoacidic phospholipids (Petit et al., 1992),

Abbreviations: CIP, ciprofloxacin; CL, cardiolipin; NAO, 10-N-nonyl acridine orange; UTI, urinary tract infection. can be used to distinguish between CL and other negatively charged lipids and to detect the presence of CL domains in bacterial membranes. Such domains play a key role in the organization of bacterial membranes, particularly in the binding of protein complexes involved in cellular processes such as cell division or membrane transport (Mileykovskaya et al., 2009; Romantsov et al., 2007).

Antibiotics exert their bactericidal effects in many ways, which can be divided into two general categories: membrane permeabilization/disruption, and inhibition of various intracellular processes (e.g. DNA replication). It is commonly accepted that the fluoroquinolone ciprofloxacin (CIP) owes most of its antibacterial properties to the inhibition of bacterial DNA topoisomerases: DNA gyrase (the preferential target in Gram-negative bacteria) and topoisomerase IV (affected mainly in Gram-positive bacteria) (Tamayo et al., 2009; Drlica \& Zhao, 1997). Binding of fluoroquinolones to the DNA topoisomerase causes inhibition of cell growth. They induce DNA double-strand breaks (Tamayo et al., 2009), and/or block DNA replication and RNA transcription. Exposure of bacteria to sub-MIC doses of CIP induces SOSresponse-controlled DNA repair (Dougherty \& Saukkonen, 1985) and cell filamentation (Mason et al., 1995; Wojnicz et al., 2007). At CIP concentrations exceeding the MIC, DNA fragmentation is rapid and leads to cell death (Drlica et al., 2008). It has also been suggested that this antibiotic, as well as other quinolones, might induce permeability changes and affect the integrity of bacterial membrane (Dougherty \& Saukkonen, 1985); however, this aspect of CIP activity has 
not been extensively studied. Thus CIP is considered as a compound preferentially acting at the DNA level. On the other hand colistin (polymyxin E) is widely recognized as a membrane-active peptide antibiotic (Mortensen et al., 2009). The main mechanism of polymyxin action is attributed to the induction of membrane permeabilization due to the destabilization of the lipid bilayer (Miller et al., 1978). Since colistin molecules bear a positive charge, anionic lipids are the natural target for their action. Colistin interacts electrostatically with the outer membrane of Gram-negative bacteria and causes release of lipopolysaccharides (Kwa et al., 2008; Mortensen et al., 2009). It has been proposed that the bactericidal activity of polymixins involves the formation of molecular contacts between the inner and outer lipid layer of the outer membrane, which may induce lipid exchange and result in loss of composition specificity. Then these compounds reach the periplasmic space and interact with the cytoplasmic membrane, causing an increase of the membrane permeability (Mortensen et al., 2009).

The completely different primary targets of CIP and colistin in bacterial cells prompted us to use these compounds to test whether NAO staining of Escherichia coli membranes could be used to detect and compare the possible effects of these antibiotics at the membrane level.

\section{METHODS}

Bacterial strains and growth conditions. We used E. coli cultures from the urine of patients suffering from urinary tract infections (UTI), isolated and identified in the Laboratory of Microbiology of the Academic Clinical Hospital, Wrocław. Cells were grown in standard liquid Mueller-Hinton broth (MHB). Fresh overnight cultures were diluted $1: 10$ in fresh MHB supplemented or not (control) with $3 / 4,1 / 2$ or $1 / 16$ MIC of colistin (500000 units $\mathrm{ml}^{-1}$, Polfa Tarchomin) or ciprofloxacin (Proxacin 1\%, WZF Polfa) and further incubated at $37{ }^{\circ} \mathrm{C} / 150$ r.p.m. to an $\mathrm{OD}_{600}$ of $0.2-0.6$.

MIC determination. MICs were determined using the standard microdilution method recommended by the Clinical and Laboratory Standards Institute (formerly National Committee for Clinical Laboratory Standards) (CLSI, 2006).

NAO staining of the cells. The stock solution of NAO (Invitrogen) in DMSO was added to the cell culture kept at $37{ }^{\circ} \mathrm{C}$ in amounts chosen to reach a final dye concentration of $400 \mathrm{nM}$. In control experiments 0.004 vol. DMSO was added to 1 vol. cell culture and no effect on bacterial viability was observed. After incubation at room temperature for $60 \mathrm{~min}$ in darkness, the cells were washed three times with sterile potassium phosphate-buffered saline (PBS) and fixed on poly-L-lysine-covered microscope slides (Polysine slides, Thermo Scientific). Two or three independent repeats were performed for each experiment.

Fluorescence microscopy and cell morphology. Images were obtained using a Nikon Eclipse E400 fluorescence microscope equipped with a planar $(\times 100)$ objective. Green and red NAO fluorescence was observed using FITC and rhodamine filters, respectively. Data acquisition was performed by NIS ELEMENTS BR 3.0 software (Nikon). Cell length was measured using the tool available in this software. About 300 cells on each image were measured. Three images were analysed for each experiment.
For determination of cell morphology the samples were Gram-stained and observed with a Nikon Eclipse E400 microscope.

Image deconvolution. Images recorded by fluorescence microscopy were stored on the hard disk and deconvoluted off-line using MacBiophotonics ImageJ software. Tikhonov-Miller or RichardsonLucy algorithms implemented in the DeconvolutionLab plugin were used to prepare the deconvoluted images presented in this paper.

Statistical analysis. Data in plots are presented as means \pm SD. The significance of differences between mean values was determined using ANOVA. $P<0.05$ was considered to be significant.

\section{RESULTS}

\section{Distribution of $\mathrm{CL}$ domains in untreated $E$. coli cells}

In preliminary experiments we checked the distribution of CL domains in the untreated E. coli cells. As presented in Fig. 1 we found that the domains present in the membranes generally displayed five patterns, occurring with different frequencies. The most frequent ( $78 \%$ of the observed cells) were cells with CL domains located exclusively at both cell poles (type 1, T1). We also found bacteria with additional septal CL location (type 3, T3), which constituted $13 \%$ of the cells (Fig. 1a, b). As well as these most frequent patterns of CL location we also detected two other domain morphologies: with CL located exclusively in the septal region (type 2, T2) or in one of the cell poles (type 4, T4). Percentages of these cell types were similar and their values were approximately $2 \%$ or $4 \%$, for $\mathrm{T} 2$ and $\mathrm{T} 4$, respectively

(a)

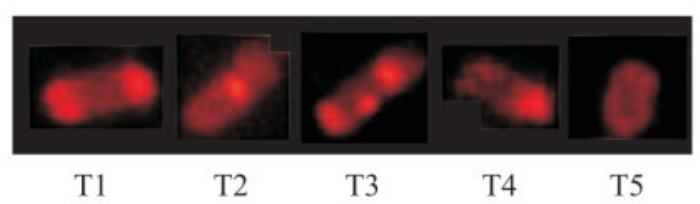

(b)

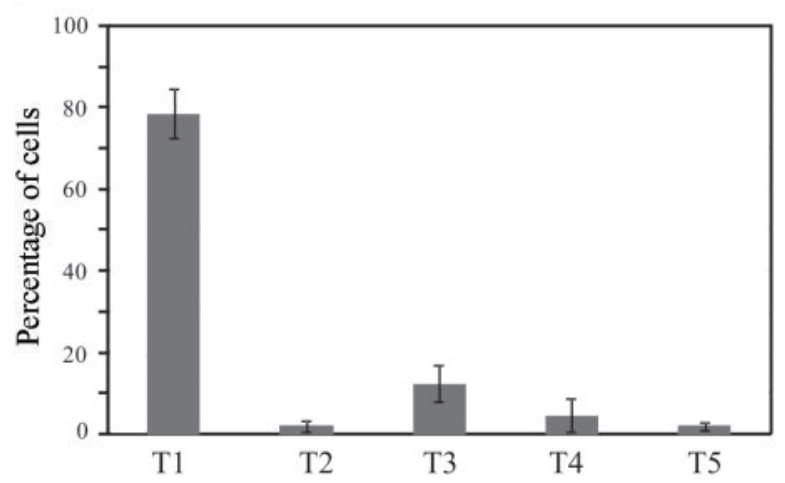

Fig. 1. Distribution patterns of $C L$ domains in E. coli unexposed to any antimicrobial agents. (a) Micrograph of red fluorescence domains. (b) Frequency (as a percentage of the total cell population) of cells with the different $\mathrm{CL}$ domain distribution patterns. 
(Fig. 1a, b). Furthermore we also observed a few E. coli cells $(2 \%)$ with no apparent CL domains in the membranes (type 5, T5).

The average length of normal E. coli cells lies in the range 2$5 \mu \mathrm{m}$. In our case the maximal length of the majority of the cells was about $3 \mu \mathrm{m}$. Surprisingly, among these untreated bacteria some filamentous cells appeared, whose length significantly exceeded $3 \mu \mathrm{m}$. They constituted only about $0.65 \%$ of the observed cells. Considering the CL distribution, we observed three different types of these filaments (Fig. 2). As well as cells with CL domains located at both cell poles (filamentous type 3, FT3), cells with spot-like CL distribution (filamentous type 2, FT2) also appeared. We also observed filaments with densely dispersed green fluorescent dot-like domains on the whole cell surface (filamentous type 1, FT1). Because these dots were not visible as red fluorescence, they were probably composed of phosphatidylglycerol but without or with very low level of CL (Fig. 2d-f). Frequencies of all three types of filaments were similar (see inset in Fig. 3a for details).

\section{Neither colistin nor CIP changes the morphology or distribution pattern of CL domains in normal- length $E$. coli cells}

In preliminary experiments we determined the MIC for CIP and colistin as $0.007 \mu \mathrm{g} \mathrm{ml}^{-1}$ and 2-4 $\mu \mathrm{g} \mathrm{ml} \mathrm{m}^{-1}$, respectively. To investigate whether CIP or colistin may cause changes in the morphology and/or distribution pattern of CL domains in E. coli membranes, we treated the cells with $3 / 4,1 / 2$ and 1/16 MICs of these drugs. No significant changes in the morphology and/or distribution pattern of CL domains were seen for normal-length cells, after either colistin or CIP treatment; the same results were obtained when subinhibitory concentrations of other drugs were used (data not shown). However, in comparison to untreated $E$. coli, we observed a significantly lower frequency of the T1 and T3 cells after CIP treatment: $35 \%$ and $5.5 \%$ respectively (Fig. 3a). Additionally, the presence of this drug resulted in a pronounced increase in the proportion of filamentous cells, as described in the next section. Gram staining indicated that the filaments observed were not ghost cells (Fig. 2g).

\section{CIP significantly increases the frequency of all types of the filamentous cells}

It is generally known that treatment of E. coli with CIP causes the formation of filamentous cells (Wojnicz et al., 2007). Also in our experiments we observed that treatment of cells isolated from the urine of patients suffering from UTI with sub-MIC doses of CIP resulted in a marked increase in the percentage of filamentous cells $(33 \%$ for cells treated with $1 / 2$ MIC of CIP). Unlike the case of the untreated cells, the frequencies of the three types of filaments described above were not equal. The largest increase was recorded for FT2 cells, which constituted about $20 \%$ of the observed cells (see inset in Fig. 3a). Cells with dot-like dispersed green fluorescent anionic phospholipid domains (FT1) appeared rarely (1.5\%), while the FT3 pattern was found in $10 \%$ of observed cells.

\section{DISCUSSION}

Staining by the fluorescent dye NAO is a relatively easy and effective technique that can be used to visualize CL distribution in bacterial membranes. An important feature of NAO is that when it is bound to anionic phospholipids
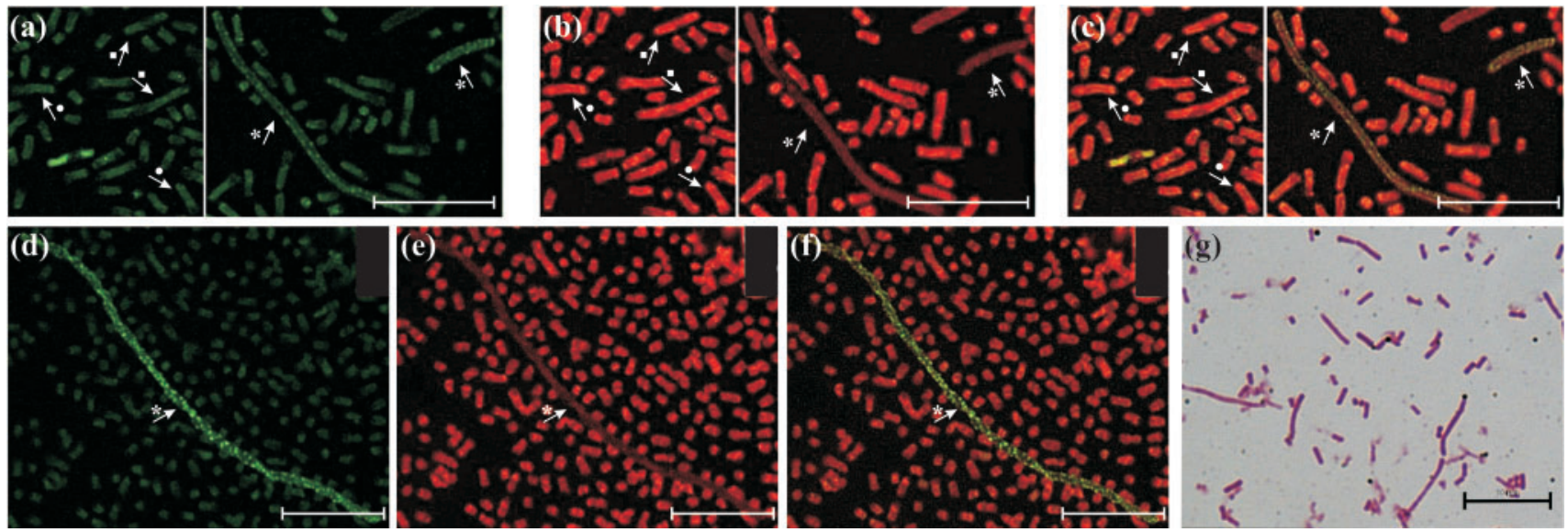

Fig. 2. Micrographs showing the distribution patterns of anionic phospholipids in cell membranes of filamentous cells. $(\mathrm{a}-\mathrm{c})$ Cells after exposure to 1/2 MIC of CIP. (d-f) An example of an FT1 filament among unexposed E. coli. Arrows with asterisks, squares and circles indicate FT1, FT2 and FT3 filaments, respectively. Deconvoluted images in (a) and (d) were made using FITC and in (b) and (e) using rhodamine filters. Panels (c) and ( $f$ ) are overlays of the images in (a-b) and (d-e), respectively. Micrograph (g) shows the morphological modifications of the cells after CIP treatment (Gram stain). Scale bars, $10 \mu \mathrm{m}$. 
(a)

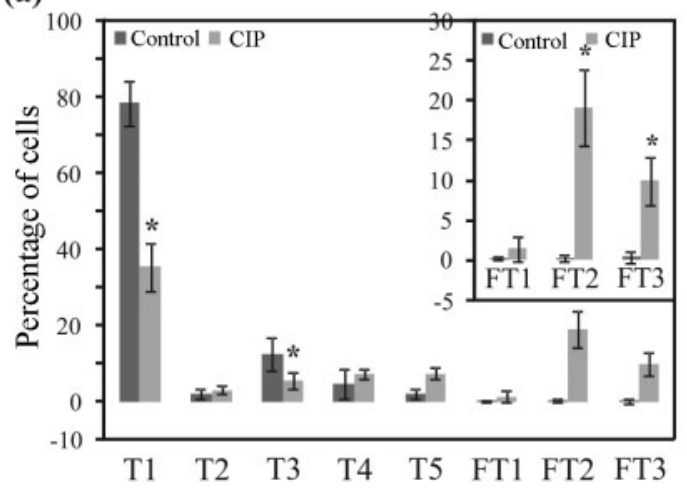

(b)

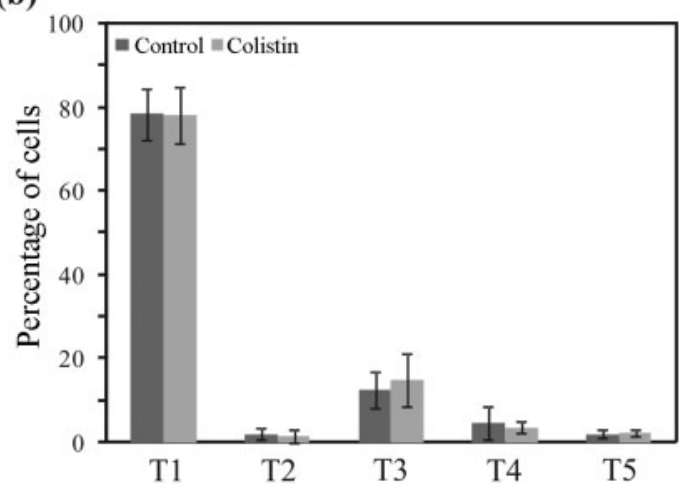

Fig. 3. Frequency of cells with different patterns of CL domain distribution, after exposure to (a) $1 / 2$ MIC of CIP and (b) $1 / 2 \mathrm{MIC}$ of colistin, compared to untreated cells. The inset in (a) shows an expanded view of the frequency of filament types among strains unexposed and exposed to $1 / 2 \mathrm{MIC}$ of CIP. Asterisks $\left(^{*}\right)$ indicate statistically significant changes $(P<0.05)$.

it generates a green fluorescence, but association of this dye with CL molecules results in the shift of its fluorescence to red (Lobasso et al., 2009). This effect is caused by the dimerization of the dye molecules after binding to CL, which has a double negative charge (Petit et al., 1992, 1994). Staining of bacterial membranes with NAO has shown that in non-dividing cells CL localizes at the poles of E. coli (Mileykovskaya \& Dowhan, 2000) and Bacillus subtilis (Kawai et al., 2004), presumably to provide the proper curvature of these cell regions and organization of proteins which regulate cell division (Mileykovskaya \& Dowhan, 2005). As CL forms domains at the septum to organize the cell division machinery (Epand \& Epand, 2009; Matsumoto et al., 2006), the cells preparing for division also show the presence of CL domains in septa. Other, unusual kinds of localization of CL domains (such as found by us: T2, T4, T5, FT1, FT2 and FT3) might presumably occur in cells which are in the late phase of division or in cells in which the division process is perturbed. In our opinion the low percentage of untreated cells showing an unusual location (T2, T4 and T5) of CL domains supports the above explanation.

The visualization of CL domains using NAO was applied in the present work to compare the effects exerted by subinhibitory concentrations of CIP and colistin on the distribution and morphology of these domains in membranes of E. coli strains isolated from the urine of patients suffering from UTI. Keeping in mind the primary effects exerted by these antibiotics on bacteria, we expected to find alterations of the CL domains after treatment of the cells with colistin, but surprisingly, changes were found exclusively in CIP-treated cells. In this case we observed an increase in the number of filamentous cells followed by alterations of the morphology and distribution of the CL domains. It is significant that among the cells exposed to CIP, the highest increase (in comparison to untreated cells) was recorded for FT2 filaments. As the fluoroquinolones inhibit DNA synthesis and disturb the cell division process, production of filamentous cells is in agreement with the mechanism of CIP action (Dougherty \& Saukkonen, 1985; Wojnicz et al., 2007).

The results of our study clearly indicate that in spite of the mode of action of colistin, subinhibitory concentrations of this polymyxin exert no effects on the distribution and morphology of CL domains in E. coli cells. Therefore we can also conclude that presumably the perturbation of membrane structure induced by colistin has a strictly local character, and that at the level of lipid domains this drug is inactive. As already mentioned, we noticed distinct perturbation and/or unusual distribution of CL domains in the filamentous cells that emerged after the CIP treatment. It is interesting that exposure of E. coli cells to sub-MIC doses of CIP especially increased the percentage of filaments with spot-like CL domains. We are not the first to report that DNA topoisomerase inhibition can be followed by the alterations at the level of the bacterial membrane. Dougherty \& Saukkonen (1985) showed that inhibition of DNA synthesis by nalidixic acid, a DNA gyrase inhibitor, results in morphological changes consistent with a loss of membrane integrity and leakage of intracellular components. Similar results were presented by Wickens et al. (2000), who noticed a decrease of both membrane integrity and membrane potential after exposure of E. coli to CIP. One of the proposed explanations of this finding is that, as a result of processes induced by inhibition of DNA replication, cells lose their capacity to synthesize necessary components and to maintain the proper membrane structure (Dougherty \& Saukkonen, 1985). An example of such a situation is the induction of the SOS response. SOS-induced TisB toxin synthesis and insertion into the inner membrane of $E$. coli causes membrane damage and loss of membrane potential (Unoson \& Wagner, 2008). In the case of our observations FT1 filamentous cells seem to be an example of dramatic membrane structure alteration: presence of exclusively green fluorescence suggests that CL is replaced in the 
membrane domains by other anionic lipids (presumably phosphatidylglycerol; Epand \& Epand, 2009).

It has also been reported that the unique chemical structure of CL allows for trapping of protons and lateral shuttling of them from oxidative phosphorylation complexes to ATP synthase (Haines \& Dencher, 2002). This ability determines a unique role of $\mathrm{CL}$ as a proton trap within membranes that conduct oxidative phosphorylation and thus ATP synthesis, which suggests why CL is found in membranes which pump protons, e.g. mitochondrial inner membrane and cell membrane of eubacteria (Haines \& Dencher, 2002). This specific CL function allows us to speculate that presumably after the fluoroquinolone treatment bacterial membranes maintain CL domains, but (due to membrane perturbation) in altered shapes. Such an effect was observed in our experiments, as CIP treatment induced a significant increase in the number of filamentous cells with FT2 morphology.

In this paper we have shown that treatment of $E$. coli by CIP causes significant morphological changes at the level of the bacterial membrane. These alterations were visualized as a change in the pattern of CL domains and we believe they are the result of the impairment of cell systems induced by the action of the antibiotic. These surprising results led us to conclude that simple fluorescent labelling might provide valuable information about membrane projection of the complex mechanisms involved in antibiotic action. We can also conclude that NAO staining of bacterial membranes is a relatively simple and efficient method of monitoring the effects exerted by different factors that do not exclusively perturb the membranes of the cells.

\section{ACKNOWLEDGEMENTS}

This work was supported by a grant from the Wroclaw Medical University, Wroclaw, Poland.

\section{REFERENCES}

CLSI (2006). Performance Standards for Antimicrobial Susceptibility Testing, 16th edn, Informational Supplement M100-S16. Wayne, PA: Clinical and Laboratory Standards Institute.

Dougherty, T. J. \& Saukkonen, J. J. (1985). Membrane permeability changes associated with DNA gyrase inhibitors in Escherichia coli. Antimicrob Agents Chemother 28, 200-206.

Drlica, K. \& Zhao, X. (1997). DNA gyrase, topoisomerase IV, and the 4-quinolones. Microbiol Mol Biol Rev 61, 377-392.

Drlica, K., Malik, M., Kerns, R. J. \& Zhao, X. (2008). Quinolonemediated bacterial death. Antimicrob Agents Chemother 52, 385-392.

Epand, R. M. \& Epand, R. F. (2009). Lipid domains in bacterial membranes and the action of antimicrobial agents. Biochim Biophys Acta 1788, 289-294.

Haines, T. H. \& Dencher, N. A. (2002). Cardiolipin: a proton trap for oxidative phosphorylation. FEBS Lett 528, 35-39.
Kawai, F., Shoda, M., Harashima, R., Sadaie, Y., Hara, H. \& Matsumoto, K. (2004). Cardiolipin domains in Bacillus subtilis Marburg membranes. J Bacteriol 186, 1475-1483.

Kwa, A. L., Tam, V. H. \& Falagas, M. E. (2008). Polymyxins: a review of the current status including recent developments. Ann Acad Med Singapore 37, 870-883.

Lobasso, S., Saponetti, M. S., Polidoro, F., Lopalco, P., Urbanija, J., Kralj-Iglic, V. \& Corcelli, A. (2009). Archaebacterial lipid membranes as models to study the interaction of 10- $\mathrm{N}$-nonyl acridine orange with phospholipids. Chem Phys Lipids 157, 12-20.

Mason, D. J., Power, E. G., Talsania, H., Phillips, I. \& Gant, V. A. (1995). Antibacterial action of ciprofloxacin. Antimicrob Agents Chemother 39, 2752-2758.

Matsumoto, K., Kusaka, J., Nishibori, A. \& Hara, H. (2006). Lipid domains in bacterial membranes. Mol Microbiol 61, 1110-1117.

Mileykovskaya, E. \& Dowhan, W. (2000). Visualization of phospholipid domains in Escherichia coli by using the cardiolipin-specific fluorescent dye 10-N-nonyl acridine orange. J Bacteriol 182, 11721175.

Mileykovskaya, E. \& Dowhan, W. (2005). Role of membrane lipids in bacterial division-site selection. Curr Opin Microbiol 8, 135-142.

Mileykovskaya, E., Ryan, A. C., Mo, X., Lin, C. C., Khalaf, K. I., Dowhan, W. \& Garrett, T. A. (2009). Phosphatidic acid and $\mathrm{N}$ acylphosphatidylethanolamine form membrane domains in Escherichia coli mutant lacking cardiolipin and phosphatidylglycerol. J Biol Chem 284, 2990-3000.

Miller, I. R., Bach, D. \& Teuber, M. (1978). Effect of polymyxin B on the structure and the stability of lipid layers. J Membr Biol 39, 49-56.

Mortensen, N. P., Fowlkes, J. D., Sullivan, C. J., Allison, D. P., Larsen, N. B., Molin, S. \& Doktycz, M. J. (2009). Effects of colistin on surface ultrastructure and nanomechanics of Pseudomonas aeruginosa cells. Langmuir 25, 3728-3733.

Petit, J. M., Maftah, A., Ratinaud, M. H. \& Julien, R. (1992). 10- $N$-nonyl acridine orange interacts with cardiolipin and allows the quantification of this phospholipid in isolated mitochondria. Eur J Biochem 209, 267-273.

Petit, J. M., Huet, O., Gallet, P. F., Maftah, A., Ratinaud, M. H. \& Julien, R. (1994). Direct analysis and significance of cardiolipin transverse distribution in mitochondrial inner membranes. Eur J Biochem 220, 871-879.

Romantsov, T., Helbig, S., Culham, D. E., Gill, C., Stalker, L. \& Wood, J. M. (2007). Cardiolipin promotes polar localization of osmosensory transporter ProP in Escherichia coli. Mol Microbiol 64, 1455-1465.

Tamayo, M., Santiso, R., Gosalvez, J., Bou, G. \& Fernández, J. L. (2009). Rapid assessment of the effect of ciprofloxacin on chromosomal DNA from Escherichia coli using an in situ DNA fragmentation assay. BMC Microbiol 9, 69.

Tucker, A. N. \& White, D. C. (1970). Heterogeneity of phospholipid composition in the bacterial membrane. J Bacteriol 102, 508-513.

Unoson, C. \& Wagner, E. G. (2008). A small SOS-induced toxin is targeted against the inner membrane in Escherichia coli. Mol Microbiol 70, 258-270.

Wickens, H. J., Pinney, R. J., Mason, D. J. \& Gant, V. A. (2000). Flow cytometric investigation of filamentation, membrane patency, and membrane potential in Escherichia coli following ciprofloxacin exposure. Antimicrob Agents Chemother 44, 682-687.

Wojnicz, D., Kłak, M., Adamski, R. \& Jankowski, S. (2007). Influence of subinhibitory concentrations of amikacin and ciprofloxacin on morphology and adherence ability of uropathogenic strains. Folia Microbiol (Praha) 52, 429-436. 